\title{
Clinical application of a special postoperative position cushion for patients after percutaneous nephrolithotomy: a randomized trial
}

\author{
Juan Xue ${ }^{1,2}$, Qun Yang ${ }^{3,4}$ \\ ${ }^{1}$ The Third Xiangya Hospital of Central South University, Key Laboratory of Medical Information Research (Central South University), College of \\ Hunan Province, Changsha, China; ${ }^{2}$ Nursing Department of the Third Xiangya Hospital of Central South University, Changsha, China; ${ }^{3}$ Clinical \\ Nursing Teaching and Research Section, the Second Xiangya Hospital, Central South University, Changsha, China; ${ }^{4}$ Department of Oncology, the \\ Second Xiangya Hospital, Central South University, Changsha, China \\ Contributions: (I) Conception and design: J Xue; (II) Administrative support: J Xue; (III) Provision of study materials or patients: J Xue; (IV) \\ Collection and assembly of data: Q Yang; (V) Data analysis and interpretation: Q Yang; (VI) Manuscript writing: All authors; (VII) Final approval of \\ manuscript: All authors. \\ Correspondence to: Qun Yang. Clinical Nursing Teaching and Research Section, The Second Xiangya Hospital, Central South University, No. 139 \\ Middle Renmin Road, Changsha 410011, China. Email: yangqun1977@csu.edu.cn.
}

\begin{abstract}
Backgroundk Percutaneous nephrolithotomy (PCNL), a minimally invasive technique, has gradually replaced traditional open surgery in the treatment of upper urinary tract stones. The aim of this study was to observe the effects of a new special position cushion for PCNL in the clinical application.

Methods: Four-hundred and fifty patients (229 males and 221 females, at the age of $47.03 \pm 8.9$ years) after PCNL were randomly divided into two groups: the control group and the experimental group ( $n=225$ /each group). Patients in the control group were treated with conventional hip sponge pads to prevent from pressure ulcers after PCNL, and patients in the experimental group used the postoperatively special position cushion which included a sacrococcygeal pad and a back pad. We observed and compared patient's position comfort, the incidence of renal hemorrhage, removal rate of the renal fistula, folding rate of the renal fistula and incidence of refractory pressure ulcer between two groups.

Results: Compared to the control group, the position comfort of the experiment group was improved. The incidence of renal hemorrhage due to improper postoperative position (hereinafter referred to as the incidence of renal bleeding), the rate of devascularization of the renal fistula, the rate of tube folding of the renal fistula, and the incidence of postoperative pressure ulcers in the experiment group were decreased in comparison with the control group, with significant differences $(\mathrm{P}<0.05)$.

Conclusions: The special position cushion increases the patient's comfort, improves the fix effect of the renal fistula, and effectively reduces the occurrence of adverse reactions such as bleeding and pressure sore caused by improper PCNL postoperative position.
\end{abstract}

Keywords: Special position pad; percutaneous nephrolithotomy (PCNL); clinical application

Submitted Oct 22, 2019. Accepted for publication Nov 02, 2020.

doi: 10.21037/apm-19-404

View this article at: http://dx.doi.org/10.21037/apm-19-404

\section{Introduction}

Urinary calculi are common urological diseases, with a $2 \%$ to $3 \%$ incidence rate, and may cause the severe hydronephrosis and even uremia. Percutaneous nephrolithotomy (PCNL) is a minimally invasive technique for renal stone removal but can cause renal parenchymal injury (1). Patients in PCNL were usually in the prone position for establishment of a surgical access from the skin to the kidneys in the upper and lower lumbar ribs and the posterior line of the scapula to the lower scapula. The nephoscopy is placed in the kidney through 
this channel, and then laser and pneumatic trajectory are used to crush and remove kidney stones. There are certain complications after PCNL, of which bleeding is the most common symptom (2). Therefore, after the clinical upswing of PCNL, patients need to take a bed rest for 5-7 days depending on the color change of the renal fistula tube drainage (3). Due to the need to lie flat and not to turn over, the incidence of pressure ulcers is increased. To prevent the kidney from bleeding, it is generally not suggested to use an air mattress with the massage function for pressure ulcer prevention in patients after PCNL. To avoid pressure ulcers, the hip sponge pads are usually used to raise the sacrococcygeal region and suspend the back, but they usually cause uncomfortable duo to the high buttocks and low back (4). Some patients do not want to use them because of the lumbosacral pain. The elevation of the buttocks also tends to move the patient's waist to force the percutaneous renal sinus, thus increasing the incidence of the postoperative renal hemorrhage. So, the current methods are not conducive to postoperative recovery. Because the renal fistula after PCNL closes to the posterior axillary line, the supine position is easy to cause the fold of the renal fistula resulting in the drainage not smooth, even the loss of the renal fistula.

To improve the current situation, in this study, a specific body position cushion for PCNL was developed, successfully applied to the clinic and achieved good results. The technology has applied for a national utility patent (No. 201420816763.9).

We present the following article in accordance with the CONSORT Reporting Checklist (available at http://dx.doi. org/10.21037/apm-19-404).

\section{Methods}

\section{Objects}

All patient experiments were approved by the Ethics Committee of the Third Xiangya Hospital, the Central South University, China (No. 2020-S030). This study was conducted in accordance with the Declaration of Helsinki (as revised in 2013). The patients signed the informed consent form and volunteered to participate in the study. From March to October 2017, patients who underwent PCNL and had a renal fistula in the Department of urology, the Second Xiangya Hospital, were recruited in this study. Four-hundred and fifty cases (229 male, 221 women) at the age of $18-76(47.03 \pm 8.9)$ years met the inclusion criteria, including multiple stones, the stone burden between 57 and $217 \mathrm{~mm}^{2}$, and the operative time between 60 and $210 \mathrm{~min}$. Patients were randomly divided into the control and experimental groups ( $\mathrm{n}=225 /$ each group): patients in the control group were treated with conventional hip sponge pads to prevent from pressure ulcers after PCNL, and patients in the experimental group used the postoperatively special position cushion with a sacrococcygeal pad and a back pad.

\section{Evaluation}

Five indicators were evaluated in patients after PCNL in two groups: (I) the position comfort rating: using visual analogue scale for indication (5), 0-2 points: comfort; 3-4 points: minor discomfort; 5-6 points: moderate discomfort; 7-8 points: severe discomfort; $9-10$ points: extreme discomfort. Evaluations were taken 3 times (in the morning, noon and evening) a day. Patients were asked to choose between different options based on their subjective feelings and the data were recorded by a staff; (II) the incidence of renal hemorrhage due to improper postoperative position: the proportion of patients with kidney bleeding caused by the stressful activity of the waist due to discomfort; (III) the deprivation rate of renal fistula: the proportion of renal fistula catheter removal after PCNL; (IV) the rate of the renal fistula folding: the proportion of the renal fistula folding after PCNL; (V) the incidence of pressure ulcers: after PCNL, the patient's Braden score was less than 9 points, or the patients met the basic conditions including instability of vital signs, heart failure, high paraplegia, forced position or limited turning under medical order. Any one of the indicators combined with extreme weight loss, high edema, and one or several items of incontinence could be reported as inevitable pressure sore.

\section{Statistical methods}

Statistical Product and Service Solutions (SPSS) 18.0 software (SPSS Inc., Chicago, IL, USA) was used for statistical analysis. The measurement data were represented by mean \pm standard deviation (SD). Two groups were tested for homogeneity of variance before the comparison. The variance was homogeneous, and nonparametric test was used.

\section{Results}

\section{Features of the new special position cushion}

The sponge pad was made of the high-density medical 


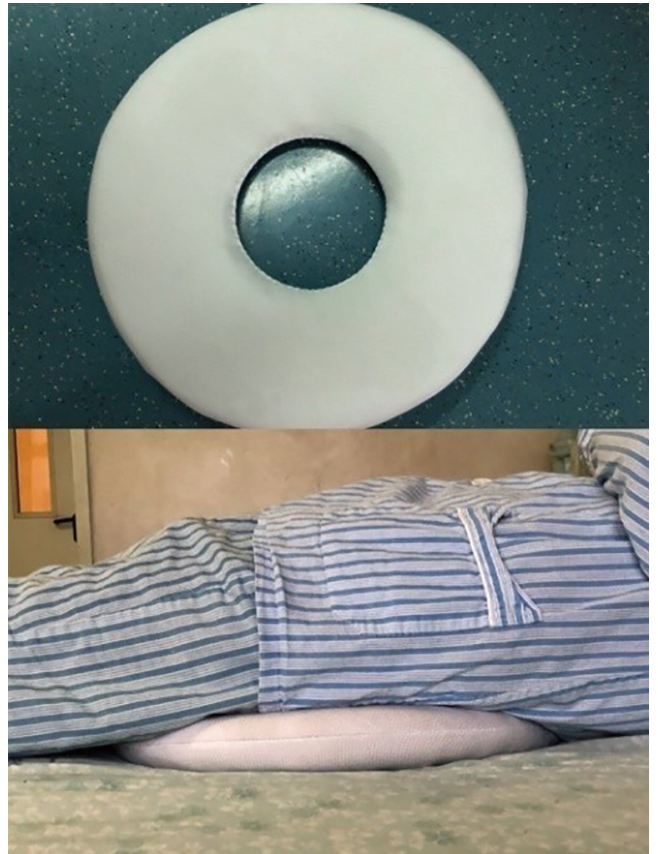

Figure 1 The conventional hip sponge pads for percutaneous nephrolithotomy. The sponge pad was made of the high-density medical sponge with a diameter of $25 \mathrm{~cm}$ and a thickness of $2.5 \mathrm{~cm}$.

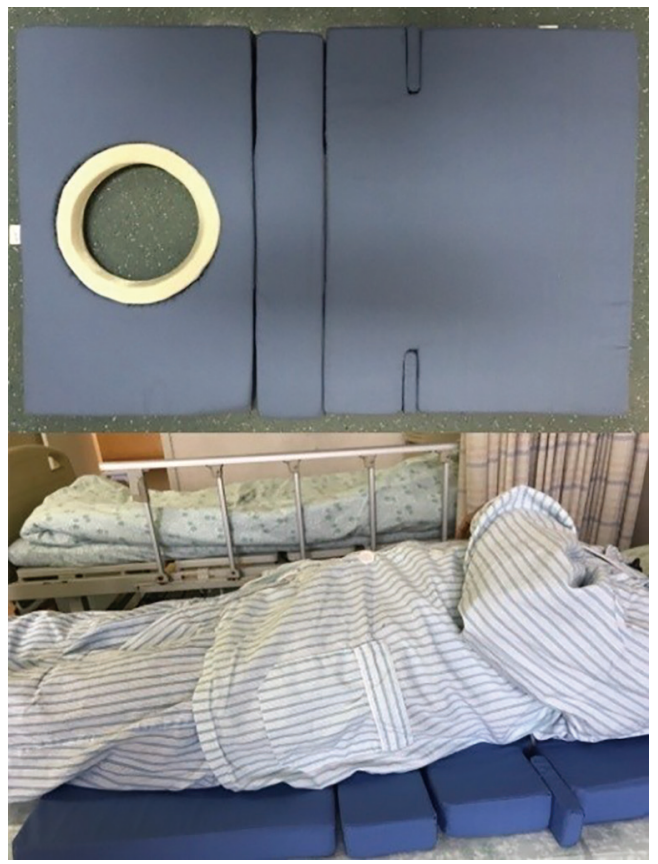

Figure 2 The special position cushion for percutaneous nephrolithotomy. The special position cushion was made of the high-density medical sponge with a width of $50 \mathrm{~cm}$, a thickness of $5 \mathrm{~cm}$ and a length of $90 \mathrm{~cm}$. sponge with a diameter of $25 \mathrm{~cm}$ and a thickness of $2.5 \mathrm{~cm}$. The pad was immediately used for the patients returning to the ward after the PCNL surgery (Figure 1). The patients in the experimental group used the new special position cushion with a sacrococcygeal pad and a back pad. The head end of the sacrococcygeal pad was connected to the tail end of the back pad. A dovetail hole was arranged in the middle portion of the sacrococcygeal pad. A tube groove was on both sides of the back pad. An adjustment pad was arranged at the joint end of the sacrococcygeal pad and the tail end of the back pad. The length of the adjustment pad could be adjusted as needed. The adjustment pad included a multi-section detachable adjustment block. The edge of the adjustment block was provided with serrated protrusions and grooves. The adjustment block was stitched together by protrusions and grooves for use of patients in different heights and sizes. The special position cushion was made of the high-density medical sponge with a width of $50 \mathrm{~cm}$, a thickness of $5 \mathrm{~cm}$ and a length of $90 \mathrm{~cm}$ (Changsha Huafeng Kangcheng Medical Instrument Co., Ltd., China) (Figure 2).

\section{Helping for patients to recovery after the PCNL by using the new special position cushion}

Results in Table 1 compared the effects of the specific position cushion (the experimental group) and the hip sponge pads (the control group) on Visual Analogue Scale (VAS) score, renal hemorrhage, renal fistula deprivation, renal fistula folding, and pressure ulcer. When $T$ test was performed for comparison of two independent samples on the VAS scores, the variance was not uniform $(\mathrm{P}=0.021<0.05)$. In the non-parametric test, the Wilcoxon $W$ value was 340.00 , the $Z$ value -5.805 , and the bilateral $\mathrm{P}=0.01$, with very significant difference. The incidence of renal hemorrhage due to improper postoperative position (hereinafter referred to as the incidence of renal bleeding), the rate of devascularization of the renal fistula, the rate of tube folding of the renal fistula, and the incidence of postoperative pressure ulcers was decreased in experiment group compared to the control group, and the differences between two groups were significant $(\mathrm{P}=0.05)$. There were no significant differences in age, gender, and educational levels between the control and experimental groups $(\mathrm{P}>0.05)$.

\section{Discussion}

Kidney stones are mineral deposits in the renal calyces and 
Table 1 Comparison of effects of the specific body position cushion and hip sponge pads

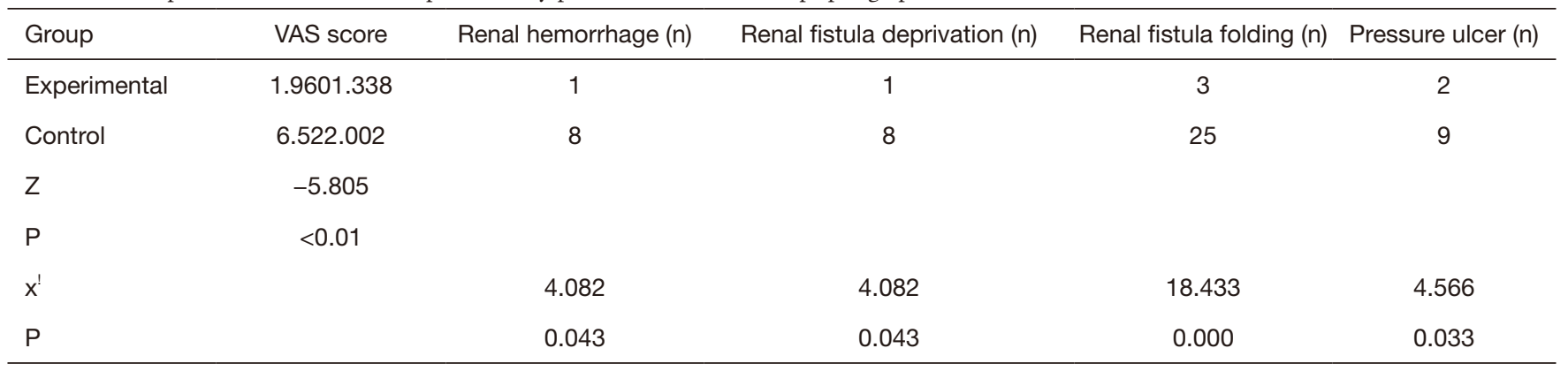

VAS, Visual Analogue Scale. $P<0.05$, significant difference; $P<0.01$, very significant difference; $P>0.05$, no significant difference.

pelvis, which are free or attached to the renal papillae. (6). About 1 in 1,000 people must be treated every year in the $\mathrm{A} \& \mathrm{E}$ or hospitalized due to kidney stone-related colic. Men are, from 5:1 to 2:1, more often affected than women (7). Since its first introduced in 1976 (8), PCNL has become a standard treatment to remove stones that are more than $2 \mathrm{~cm}$ in size and presented near the pelvic region. PCNL is a minimally-invasive procedure to remove stones from the kidney by a small puncture wound through the skin. After PCNL, patients generally need to rest in bed for 5-7 days for recovery (9), because the nephrostomy tube (NT) might leave in situ to drain the kidney, and the nephrostomy tract might tamponade (10).

Currently, there are no specifically designed body position pads for patients after the PCNL surgery on the market. In the present study, we designed a postoperatively special position cushion with an adjustable length to avoid low hip back and renal fistula discount. The special position cushion is convenient to use and helpful to recovery of patients after PCNL. The advantages of the present invention are as follows: (I) patient's postoperative position comfort is increased. The back pad is provided to avoid the phenomenon of higher hips and lower back, which effectively increases the comfort and reduces patient's suffering; (II) the incidence of renal bleeding due to improper position is effectively reduced; (III) the rates of tube removal and tube folding are effectively reduced. The special position cushion has a card tube groove that fixes the renal fistula tube on the posterior side of the patient's ankle to avoid the phenomenon that the renal fistula tube is discounted resulting in unsmooth of the flow. It also effectively prevents the occurrence of the tube removal. Therefore, the new cushion is beneficial to the recovery of postoperative patients; (IV) the incidence of inevitable pressure sores is effectively reduced, which prevents from inevitable pressure ulcers after PCNL; $(\mathrm{V})$ the special position cushion is user-friendly designed. An adjustment pad includes a plurality of detachable adjustment blocks. The length of the pad can be adjusted by setting the different number of adjustment blocks. Therefore, the overall length of the special position cushion can be adjusted after the dermatoscope to patients with different heights. In summary, the special position cushion for PCNL has solved some problems caused by existing products, which is practically valuable and worthy of clinical promotion.

There are still some limitations in this study: First, this study has time constraints, because it was taken from March to October 2017 in our hospital. Second, there lacks previous research studies on this topic, so we need to further prove the results in more patients in different hospitals.

\section{Acknowledgments}

Funding: This work was supported by the National Social Science Foundation of China (Grant No. 17ZDA037), and the Innovation Platform's Open Foundation of Education Department in Hunan Province, China (Grant No.18K004), and Provincial Department of Health, PI, General Project, China (No: C2015-86).

\section{Footnote}

Reporting Checklist: The authors have completed the CONSORT Reporting Checklist. Available at http://dx.doi. org/10.21037/apm-19-404

Data Sharing Statement: Available at http://dx.doi. org/10.21037/apm-19-404

Conflicts of Interest: All authors have completed the ICMJE 
uniform disclosure form (available at http://dx.doi. org/10.21037/apm-19-404). The authors have no conflicts of interest to declare.

Ethical Statement: The authors are accountable for all aspects of the work in ensuring that questions related to the accuracy or integrity of any part of the work are appropriately investigated and resolved. The study was approved by the Ethics Committee of the Third Xiangya Hospital, the Central South University, China (No. 2020S030). This study was conducted in accordance with the Declaration of Helsinki (as revised in 2013). The patients signed the informed consent form and volunteered to participate in the study.

Open Access Statement: This is an Open Access article distributed in accordance with the Creative Commons Attribution-NonCommercial-NoDerivs 4.0 International License (CC BY-NC-ND 4.0), which permits the noncommercial replication and distribution of the article with the strict proviso that no changes or edits are made and the original work is properly cited (including links to both the formal publication through the relevant DOI and the license). See: https://creativecommons.org/licenses/by-nc-nd/4.0/.

\section{References}

1. Yu J, Park HK, Kwon HJ, et al. Risk factors for acute kidney injury after percutaneous nephrolithotomy: Implications of intraoperative hypotension. Medicine (Baltimore) 2018;97:e11580.

Cite this article as: Xue J, Yang Q. Clinical application of a special postoperative position cushion for patients after percutaneous nephrolithotomy: a randomized trial. Ann Palliat Med 2021;10(2):1861-1865. doi: 10.21037/apm-19-404
2. Un S, Cakir V, Kara C, et al. Risk factors for hemorrhage requiring embolization after percutaneous nephrolithotomy. Can Urol Assoc J 2015;9:E594-E598.

3. Todea-Moga C, Boja R, Porav-Hodade D, et al. The Impact of comorbidities on the efficacy of percutaneous nephrolitotomy (PCNL) in elderly patients. Acta Medica Marisiensis 2016;62:243-7.

4. Bozzini G, Verze P, Arcaniolo D, et al. A prospective randomized comparison among SWL, PCNL and RIRS for lower calyceal stones less than $2 \mathrm{~cm}$ : a multicenter experience : A better understanding on the treatment options for lower pole stones. World J Urol 2017;35:1967-75.

5. Reed CC, Wolf WA, Cotton CC, et al. A visual analogue scale and a Likert scale are simple and responsive tools for assessing dysphagia in eosinophilic oesophagitis. Aliment Pharmacol Ther 2017;45:1443-8.

6. Khan SR, Pearle MS, Robertson WG, et al. Kidney stones. Nat Rev Dis Primers 2016;2:16008.

7. Bartl R, Bartl C. Kidney Stones. Bone Disorders. Springer International Publishing 2017; 561-2.

8. Fernstrom I, Johansson B. Percutaneous pyelolithotomy. A new extraction technique. Scand J Urol Nephrol 1976;10:257-9.

9. Crook TJ, Lockyer CR, Keoghane SR, et al. A randomized controlled trial of nephrostomy placement versus tubeless percutaneous nephrolithotomy. J Urol 2008;180:612-4.

10. Alyami F, Norman RW. Is an overnight stay after percutaneous nephrolithotomy safe? Arab J Urol 2012;10:367-71. 